\title{
Regulatory effect of microRNA-135a on the Th1/Th2 imbalance in a murine model of allergic rhinitis
}

\author{
YANYUN LUO*, YUQIN DENG* ${ }^{*}$ ZEZHANG TAO, SHIMING CHEN, BOKUI XIAO, JIE REN, \\ ZHE CHEN, JIBO HAN, YONGGANG KONG, YU XU and MINJIE DENG \\ Department of Otolaryngology - Head and Neck Surgery, Renmin Hospital of Wuhan University, \\ Wuhan, Hubei 430060, P.R. China
}

Received October 30, 2013; Accepted February 10, 2014

DOI: 10.3892/etm.2014.1855

\begin{abstract}
Allergic rhinitis (AR) is primarily caused by a Thelper cell (Th) $1 / \mathrm{Th} 2$ imbalance. In a murine AR model of a previous study, the serum ovalbumin (OVA)-sIgE concentration was high, whereas microRNA (miR)-135a was lowly expressed in the nasal mucosa. The abnormal expression pattern of miR-135a coincided with highly expressed endogenous factors, including GATA binding protein (GATA)-3 and interleukin (IL)-4, and lowly expressed factors, including T-box expressed in T cells (T-bet) and interferon (IFN)- $\gamma$. We hypothesized that miR-135a may play an important role in immune regulation in AR mice. In the present study, AR was induced by OVA in the mice. Two groups of the AR mice were treated with a miR-135a mimic and a mimic control, respectively. The serum and nasal mucosa were collected for analysis. Following miR-135a application, the serum OVA-sIgE concentration was significantly reduced. In the nasal mucosa, the expression levels of miR-135a were higher, the mRNA and protein expression levels of GATA-3 and IL-4 were lower, and the expression levels of T-bet and IFN- $\gamma$ were higher. The miR-135a corrected the Th1/Th2 imbalance in the AR mice. Findings of this study may provide a basis for novel genetic treatments in addressing allergic diseases.
\end{abstract}

\section{Introduction}

Allergic rhinitis (AR) is a common disease that affects the quality of life and induces other risk factors, including asthma, termed as 'one airway, one disease'. The key mechanism which causes AR is the differentiation imbalance of two T-helper cell

Correspondence to: Dr Zezhang Tao, Department of Otolaryngology - Head and Neck Surgery, Renmin Hospital of Wuhan University, 238 Jie-Fang Road, Wuhan, Hubei 430060, P.R. China

E-mail: taozz2013@126.com

*Contributed equally

Key words: allergic rhinitis, GATA-3, microRNA, mimic, T helper cell 1/T helper cell 2 imbalance
(Th) subsets, Th1/Th2, which are CD4+ helper T lymphocytes, as Th2 presents a higher immune response than Thl (1-5). T-box expressed in T cells (T-bet) and interferon (IFN)- $\gamma$ are the main Thl-specific transcription factors and cytokines, and GATA binding protein (GATA)-3 and interleukin (IL)-4 are the main Th2-specific transcription factors and cytokines. They play important roles in the differentiation of Th0 cells into Thl and Th2 cells (6-13). As GATA-3 is a Th2-specific transcription factor and plays a pivotal role in the allergic immune response, it is considered as an important therapeutic target for treating allergic diseases (7-8,10-12,14-15).

MicroRNAs (miRNAs or miRs) are a class of small molecule RNAs, which play an important role in the regulation of gene expression at the post-transcriptional level (16). MiRNAs bind to the 3' UTR (3' untranslated region) of target genes and inhibit protein translation, by regulating the expression levels of target genes (17). Numerous studies have shown that miRNA regulates proliferation, differentiation and immune function in a variety of types of immune cell within the innate and adaptive immune systems (18-38). The roles of individual types of miRNA in the immune system have also been investigated, including those of miR-21 (21-22), miR-126 (23), miR-133a (24,25), miR-145 (26), miR-146a (27), miR-150 (28), miR-155 (29-37) and miR-181a (38). Among them, only miR-21 (21), miR-126 (23) and miR-155 (34-37) were demonstrated to play a regulatory role in balancing the Th1/Th2 immune response. A deficiency in miR-21 may cause an increase in the levels of IL-12 in the allergic airway by targeting IL-12p35, an increase in the levels of IFN- $\gamma$ and a reduction in the levels of IL-4 through the IL-12/IFN- $\gamma$ pathway, thus causing divergence in enhanced cell differentiation, characteristic of a Th1 immunological reaction (21). MiR-126 upregulates the expression levels of the gene POU domain class 2 associating factor 1 . This gives rise to high expression levels of the transcription factor PU.1 and low expression levels of GATA-3, thereby inhibiting the secretion of the Th2 cytokines, IL-4, IL-5 and IL-13, in the airway of an asthma mouse model and relieving the airway allergic inflammatory reaction (23). MiR-155 has been demonstrated to act in the regulation of Thl/Th2 differentiation and proliferation. Lack of miR-155 led to the enhancement of its target transcription factor c-Maf, causing favorable differentiation of Th0 cells into Th 2 cells, with enhanced production levels of 


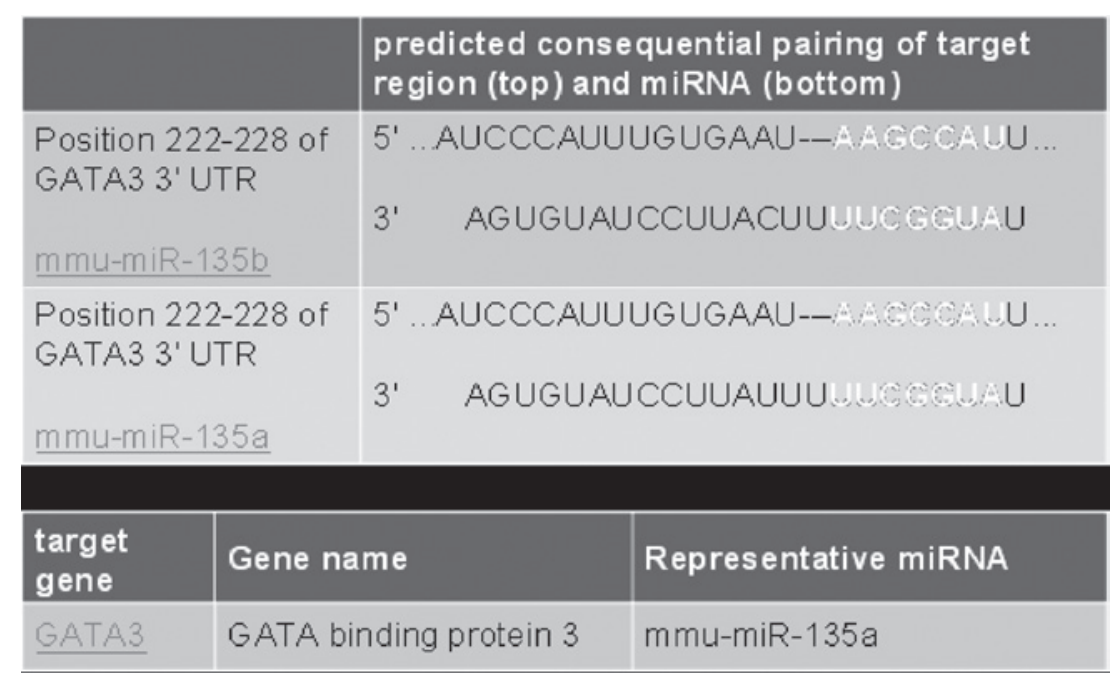

Figure 1. The predicted consequential pairing of target regions and miRNAs. Top: the predicted target region; Bottom: the predicted miRNA.

the Th2 cytokine, IL-4 (34). Lack of miR-155 also increased another target, suppressor of cytokine signaling 1 (SOCS1). The increased levels of SOCS1 protein thus inhibited IL-12 and IFN- $\gamma$ to block Th1 cell differentiation, but promoted Th2 cell differentiation (35-36). Conversely, overexpression of miR-155 caused the differentiation of Th0 cells into Th1 cells. Furthermore, repression of miR-155 expression increased the expression levels of another target, IFN- $\gamma$ receptor $\alpha$ chain $(\mathrm{R} \alpha)$, suggesting that IFN- $\gamma \mathrm{R} \alpha$ inhibited the response of Th1 cells to the antiproliferative effects of IFN- $\gamma$ (37).

In a previous study, an online bioinformatics program, which predicts and analyzes immune target genes of miRNAs, indicated that a single type of miRNA may be able to control immune regulation through differentiation divergence favoring Th2 cells via GATA-3 target (16). As yet, no studies have confirmed this prediction. Therefore, the present study analyzed the 3' UTR of mouse GATA-3 and miR-135 with TargetScan. This study may provide a basis for miRNAs to be applied in novel genetic treatments (39) for AR and other allergic diseases.

\section{Materials and methods}

Experimental animals. Specific pathogen-free male BALB/c mice, aged 5-6 weeks with a weight of 15-18 g, were purchased from the Experimental Animal Center, Renmin Hospital of Wuhan University (Wuhan, China). The animal experiments were approved by the Animal Ethics Committee, Renmin Hospital of Wuhan University. All mice were maintained under standard conventional conditions including mild light (dark), temperature $\left(18-22^{\circ} \mathrm{C}\right)$ and humidity $(50-60 \%)$, with food and water ad libitum.

Preparation of the AR mouse model. The mice were injected intraperitoneally for primary sensitization and then they were treated intranasally following primary sensitization for local stimulation and secondary immunization. The AR group mice were injected intraperitoneally with $400 \mu$ l saline containing $10 \mu \mathrm{g}$ ovalbumin (OVA) and $2 \mathrm{mg}$ aluminum hydroxide (Sigma-Aldrich, St. Louis, MO, USA) on days 1 and 8 to promote drug sensitization. Between days 15 and 21, the mice were administered intranasal drops of $20 \mu \mathrm{l}$ saline containing $200 \mu \mathrm{g}$ OVA each day to continuously challenge AR. At the same time, the control group mice were injected intraperitoneally or treated intranasally with an identical dose of saline $(400 \mu \mathrm{l}$ for sensitization on day 1 and $8 ; 20 \mu 1$ for continuous challenge each day by the same operator and transferpettor).

AR intervention by a miR-135a mimic and mimic control. The mimic and mimic control group mice were intraperitoneally sensitized and intranasally challenged with OVA, similar to that of the AR group. On days 14, 16, 18 and 20, the mimic group was treated intranasally with $20 \mu$ l saline containing $50 \mu \mathrm{g}$ miR-135a mimic to intervene with the incidence of AR, while the mimic control group was treated under identical conditions with an arbitrary sequence as previously described (23). The mimic is a cholesterol-conjugated mature miRNA mimic. The miR-135a mimic sequence was as follows: 5'-UAUGGCUUUUUAUUCCUAUGUGA-3'; and the the mimic control sequence was as follows: 5'-UUUGUACUA CACAAAAGUACUG-3' (Guangzhou RiboBio Co., Ltd., Guangzhou, China).

Detection of the serum OVA-sIgE concentration by an ELISA. Blood was extracted from the orbital venous plexus of the anesthetized mice $24 \mathrm{~h}$ after the last nasal cavity challenge. The blood was left to stand for $20 \mathrm{~min}$ prior to $10 \mathrm{~min}$ centrifugation at $700 \mathrm{x} \mathrm{g}$. The upper serum was collected and stored at $-20^{\circ} \mathrm{C}$. The serum concentration of OVA-sIgE was detected by an ELISA (R\&D Systems, Minneapolis, MN, USA).

TargetScanMouse prediction. TargetScanMouse, an online bioinformatics program, was used analyze the 3' UTR of GATA-3 and the miR-135a 3' UTR, and thus to predict the consequential pairing of the target region and miRNA (Fig.1) according to the method described previously (16).

Quantitative polymerase chain reaction ( $q P C R)$. The anesthetized mice were euthanized by cervical dislocation under 
A

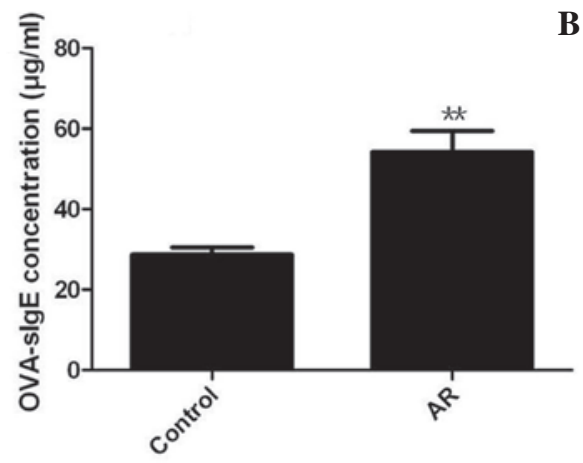

B

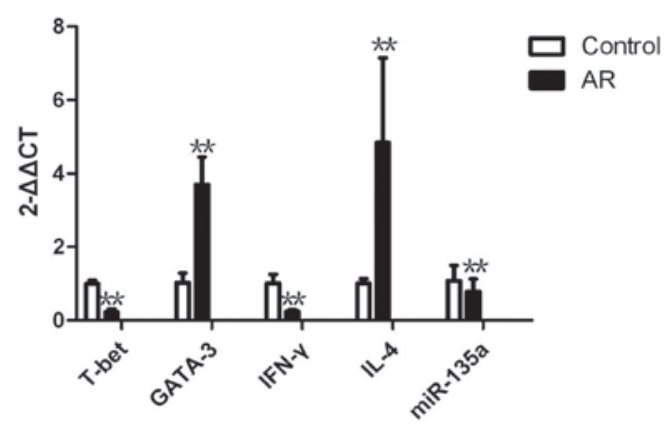

Figure 2. Expression levels of miR-135a in the nasal mucosa of AR mice. AR was induced in the mice by intraperitoneal injection and intranasal drops of OVA. (A) The serum OVA-sIgE concentrations were detected in the AR and control groups by an ELISA $24 \mathrm{~h}$ after the last nasal cavity challenge. (B) SYBR Green qPCR was used to assay the mRNA expression levels of T-bet, GATA-3, IFN- $\gamma$ and IL-4 in the nasal mucosa of the two groups. Bulge-Loop ${ }^{\mathrm{TM}}$ miRNA qPCR was used to assay for the relative expression levels of miR-135a of the nasal mucosa in the two groups. ( $\mathrm{n}=10-20$ mice/group), ${ }^{* *} \mathrm{P}<0.01$. Data were averaged by two independent experiments. OVA, ovalbumin; AR, allergic rhinitis; T-bet, T-box expressed in T cells; GATA, GATA binding protein; IFN, interferon; IL, interleukin; miR, microRNA; qPCR, quantitative polymerase chain reaction.

A

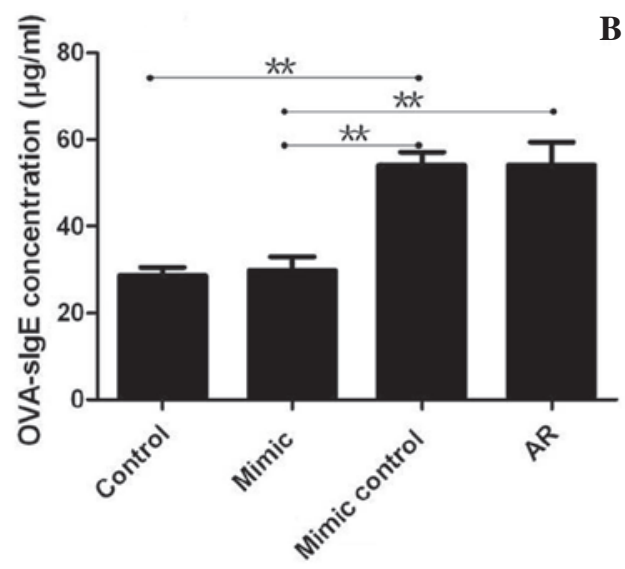

C

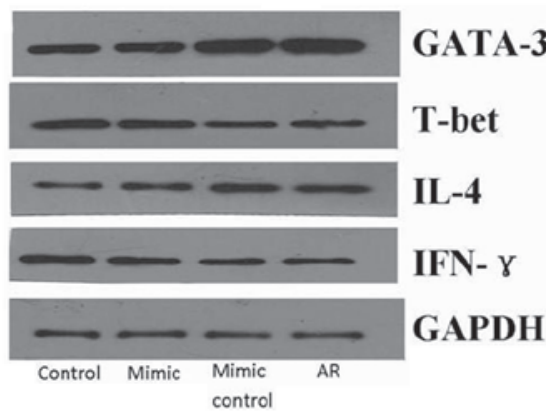

D
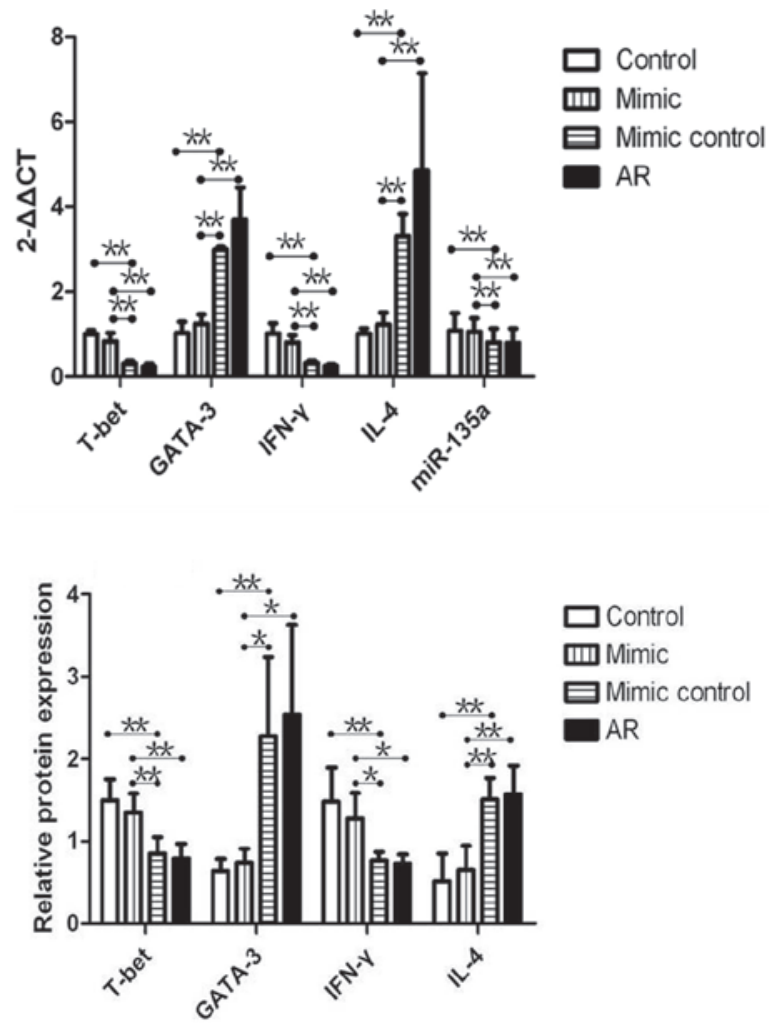

Figure 3. Regulatory effect of a miR-135a mimic on AR. The mimic and mimic control group mice were intraperitoneally sensitized and intranasally challenged by OVA, similar to that of the AR group. (A) The serum OVA-sIgE concentrations were detected by an ELISA in the control, mimic, mimic control and AR groups $24 \mathrm{~h}$ after the last nasal cavity challenge. (B) In the nasal mucosa of the four groups, Bulge-Loop ${ }^{\mathrm{TM}}$ miRNA qPCR was used to detect the relative miR-135a expression levels; and SYBR-Green qPCR was used to assay for the relative mRNA expression levels of T-bet, GATA-3, IFN- $\gamma$ and IL-4. (C-D) Western blot analysis was used to detect the relative protein expression levels of T-bet, GATA-3, IFN- $\gamma$ and IL-4. (n=10-20 mice/group), ${ }^{*} \mathrm{P}<0.05$, ${ }^{* *} \mathrm{P}<0.01$. Data were averaged by two independent experiments. OVA, ovalbumin; AR, allergic rhinitis; T-bet, T-box expressed in T cells; GATA, GATA binding protein; IFN, interferon; IL, interleukin; miR, microRNA; qPCR, quantitative polymerase chain reaction.

sterile conditions. The center seam of the nose from the vertical hard palate of each mouse was excised and sectioned. The nasal septum and mucous membrane of each bilateral nasal cavity lateral wall was eviscerated and placed into sterile vials for storage in liquid nitrogen. The RNA extraction with TRIzol (Invitrogen, Carlsbad, CA, USA) was performed strictly according to the manufacturer's instructions, and the product was treated with DNase digestion. In the nasal mucosa of the control, mimic, mimic control and AR groups, Bulge-Loop ${ }^{\mathrm{TM}}$ miRNA qPCR was used to detect the miR-135a expression levels, and U6 small nuclear RNA was used as an internal control (Guangzhou RiboBio Co., Ltd.). SYBR-Green qPCR was used to detect the relative mRNA expression levels of T-bet, GATA-3, IFN- $\gamma$ and IL-4. $\beta$-actin was used as an 
internal control (Fermentas, Waltham, MA, USA). The qPCR results used $2^{-\triangle \Delta C T}$ to represent the relative mRNA expression levels of the target genes. The primer sequences used were: F, 5'-CCTGGACCCAACTGTCAACT-3' and R, 5'-AACTGT GTTCCCGAGGTGTC-3' for T-bet; F, 5'-CTGGAG GAGGAACGCTAATG-3' and R, 5'-AGATGTGGCTCA GGGATGAC-3' for GATA-3; F, 5'-ACTGGCAAAAGGATG GTGAC-3' and R, 5'-GACCTGTGGGTTGTTGACCT-3' for IFN- $\gamma$; F, 5'-TCAACCCCCAGCTAGTTGTC-3' and R, 5'-AAATATGCGAAGCACCTTGG-3' for IL-4; F, 5'-CAC GATGGAGGGGCCGGACTCATC-3' and R, 5'-TAAAGA CCTCTATGCCAACAC AGT-3' for $\beta$-actin.

Western blotting. Western blot analysis was used to detect the relative protein expression levels of T-bet, GATA-3, IFN- $\gamma$ and IL-4 within the nasal mucosa of the control, mimic, mimic control and AR groups. The relative protein expression levels of the target genes were indicated on the gray scale ratios between each target gene and GAPDH (T-bet antibody sc-21003, GATA-3 antibody sc-9009, IFN- $\gamma$ antibody sc-59992, and IL-4 antibody sc-1260; Santa Cruz Biotechnology, Inc., Santa Cruz, CA, USA).

Statistical analysis. Data are presented as the mean \pm standard deviation. SPSS statistical software, version 17.0 (SPSS, Inc., Chicago, IL, USA) was used for statistical analysis. Differences between groups were compared using Student's $\mathrm{t}$-test or analysis of variance. $\mathrm{P}<0.05$ was considered to indicate a statistically significant difference.

\section{Results}

Low expression levels of miR-135a in the nasal mucosa of $A R$ mice. Following intraperitoneal sensitization and intranasal challenge by OVA, the AR group mice elicited symptoms similar to the clinical occurrence of AR, i.e., nose scratching, sneezing and watery nasal discharge. The control group mice elicited no symptoms. The serum OVA-sIgE concentration in the AR group was significantly elevated compared with that of the control group (Fig. 2). The mRNA expression levels of T-bet and IFN- $\gamma$ were significantly reduced and those of GATA-3 and IL-4 were significantly increased in the AR mice compared with those in the control group (Fig. 2).

By using Mouse, it was found that the sequence 5'-AAGCCAU-3' of the 3' UTR of GATA-3, at the sites of 222-228, had specific binding sites with the 3'-UUCGGUA-5' of miR-135a. It was also found that the targets of the miR-135a 3' UTR included the 3' UTR of GATA-3. The expression levels of miR-135a in the nasal mucosa of the AR mice were significantly lower than those of the control group (Fig. 2).

Low serum OVA-sIgE concentration in mice following AR intervention using a miR-135a mimic. Following intervention with a miR-135a mimic and a mimic control, it was demonstrated that the serum OVA-sIgE concentration in the mimic group was significantly lower than that in the mimic control and AR groups, but with no significant difference to that of the control group. No significant difference in the serum OVA-sIgE concentrations between the mimic control and AR groups was observed. However, the mimic control and AR groups exhibited significantly higher concentrations of serum OVA-sIgE compared with that of the control group (Fig. 3).

High $m R N A$ and protein expression levels of miR-135a and low $m R N A$ and protein expression levels of GATA-3 and $I L-4$ in the nasal mocosa following miR-135a mimic intervention. By using a miR-135a mimic and a mimic control in the nasal mucosa of the mice, the expression levels of miR-135a were significantly higher, and the expression levels of GATA-3 and IL-4 were significantly lower in the mimic group than those of the mimic control and AR groups. However, no significant difference in the levels of them between the mimic and control groups was observed. The expression levels of miR-135a, GATA-3 and IL-4 in the nasal mucosa of the mimic control group were not significantly different from those of the AR group. However, the mimic control and AR groups had significantly lower expression levels of miR-135a and higher expression levels of GATA-3 and IL-4 compared with those of the control group (Fig. 3).

High mRNA and protein expression levels of T-bet and IFN- $\gamma$ in the nasal mucosa following miR-135a mimic intervention. It was also demonstrated that the mRNA and protein expression levels of T-bet and IFN- $\gamma$ in the nasal mucosa of the mimic group were significantly higher than those of the mimic control and AR groups. Between the mimic and control groups, the difference in the levels of T-bet and IFN- $\gamma$ was not significant. The mRNA and protein expression levels of T-bet and IFN- $\gamma$ in the nasal mucosa of the mimic control group were not significantly different compared with those of the AR group. The mimic control and AR groups exhibited significantly lower expression levels than those of the control group (Fig. 3).

\section{Discussion}

AR is a disease with symptoms including an itchy nose, sneezing and watery nasal discharge. In the present study, AR mice were induced by intraperitoneal injection and intranasal OVA exposure. Compared with those of the control group, the mice in the AR group elicited symptoms of nose itching, sneezing and watery nasal discharge, along with an increase in the concentration of the serum OVA-sIgE. A previous studied showed that the mRNA expression levels of T-bet and IFN $-\gamma$ were lower, whereas the mRNA expression levels of GATA-3 and IL-4 were higher in the nasal mucosa of AR mice compared with those of normal mice, thus inhibiting the Th1 immune response and enhancing the Th2 immune response, respectively (40). The mRNA imbalance of T-bet/GATA-3 and IFN- $\gamma / \mathrm{IL}-4$ results in an imbalance of the Thl/Th2 immune response $(4,41)$, which leads to the occurrence of AR. Enhancing the Th1 and/or reducing the Th2 immune response to correct the Thl/Th2 imbalance may prevent allergic inflammatory reactions and ease the symptoms of allergic diseases (2-3,5,13-15). Regarding its important regulatory role in Th2 cell differentiation and Th2 cytokine secretion, GATA-3 is considered to be an important target for allergic diseases (7-8,10-12,14-15).

To investigate the immune regulatory effects of miRNAs on Th1/Th2 cell differentiation, a previous study used 
computer bioinformatics to forecast and analyze possible target genes of miRNAs in the immune system and identified that the Th2-specific transcription factor GATA-3 was a potential candidate, owing to its ability to combine with one or several miRNAs (16). The results indicated one or several miRNA candidates were involved in the regulation of Th2 cell differentiation through GATA-3. In the present study, it was demonstrated that GATA-3 and miR-135a were specifically binding to each other, suggesting that miR-135a may play a role in affecting the Th1/Th2 imbalance in AR mice. To test this hypothesis, a miR-135a mimic and a mimic control were used in the present study. It was demonstrated that the miR-135a mimic reduced the serum OVA-sIgE concentration, and downregulated the mRNA and protein levels of GATA-3 and IL-4. Furthermore, the miR-135a mimic caused the mRNA and protein levels of T-bet and IFN- $\gamma$ to increase in the nasal mucosa. These results further indicated that treatment with miR-135a was effective for correcting the Th1/Th2 imbalance in AR mice.

Overall, this study showed that a miR-135a mimic corrected the imbalance of Th1/Th2 in the nasal mucosa of AR mice. The mimic also reduced the serum OVA-sIgE concentration. Thus, treatment with miR-135a was effective in AR and may provide a basis for miRNA application towards novel genetic treatments for allergic diseases.

\section{Acknowledgements}

The authors would like to thank the National Natural Science Foundation of China (fund no. 81070766) for supporting this study. The authors also thank Mr. Yue Ma (School of Foreign Language and Literature, Wuhan University, Wuhan, China) for his professional review and editing of this study.

\section{References}

1. Tournoy KG, Hove C, Grooten J, Moerloose K, Brusselle GG and Joos GF: Animal models of allergen-induced tolerance in asthma: are T-regulatory-1 cells (Tr-1) the solution for T-helper-2 cells (Th-2) in asthma? Clin Exp Allergy 36: 8-20, 2006.

2. Yamamoto K, Kawamura I, Tominaga T, et al: Listeriolysin O, a cytolysin derived from Listeria monocytogenes, inhibits generation of ovalbumin-specific Th2 immune response by skewing maturation of antigen-specific $\mathrm{T}$ cells into Th1 cells. Clin Exp Immunol 142: 268-274, 2005.

3. Obihara CC, Kimpen JL and Beyers N: The potential of Mycobacterium to protect against allergy and asthma. Curr Allergy Asthma Rep 7: 223-230, 2007.

4. Yong J, Chen GQ, Huang B and Wu S: Correlation between the ratio of T-bet/GATA-3 and the levels of IL- 4 and IFN- $\gamma$ in patients with allergic asthma. Mol Med Rep 4: 663-666, 2011.

5. Park HJ, Lee CM, Jung ID, et al: Quercetin regulates Th1/Th2 balance in a murine model of asthma. Int Immunopharmacol 9 : 261-267, 2009.

6. Kim HY, Pichavant M, Matangkasombut P, et al: The development of airway hyperreactivity in T-bet-deficient mice requires CD1d-restricted NKT cells. J Immunol 182: 3252-3261, 2009.

7. Barnes PJ: Role of GATA-3 in allergic diseases. Curr Mol Med 8: 330-334, 2008.

8. Yagi R, Zhu J and Paul WE: An updated view on transcription factor GATA3-mediated regulation of Th1 and Th2 cell differentiation. Int Immunol 23: 415-420, 2011.

9. Naito T, Tanaka H, Naoe Y and Taniuchi I: Transcriptional control of T-cell development. Int Immunol 23: 661-668, 2011.

10. Paul WE: What determines Th2 differentiation, in vitro and in vivo? Immunol Cell Biol 88: 236-239, 2010.

11. Zhu J: Transcriptional regulation of Th2 cell differentiation. Immunol Cell Biol 88: 244-249, 2010.
12. Ho IC, Tai TS and Pai SY: GATA3 and the T-cell lineage: essential functions before and after T-helper-2-cell differentiation. Nat Rev Immunol 9: 125-135, 2009.

13. Wang SY, Yang M, Xu XP, et al: Intranasal delivery of T-bet modulates the profile of helper $\mathrm{T}$ cell immune responses in experimental asthma. J Investig Allergol Clin Immunol 18: 357-365, 2008.

14. Choi JR, Lee CM, Jung ID, et al: Apigenin protects ovalbumin-induced asthma through the regulation of GATA-3 gene. Int Immunopharmacol 9: 918-924, 2009.

15. Maneechotesuwan K, Yao X, Ito K, et al: Suppression of GATA-3 nuclear import and phosphorylation: a novel mechanism of corticosteroid action in allergic disease. PLoS Med 6: e1000076, 2009.

16. Asirvatham AJ, Gregorie CJ, Hu Z, Magner WJ and Tomasi TB: MicroRNA targets in immune genes and the Dicer/Argonaute and ARE machinery components. Mol Immunol 45: 1995-2006, 2008.

17. Bartel DP: MicroRNAs: target recognition and regulatory functions. Cell 136: 215-233, 2009.

18. Xiao C and Rajewsky K: MicroRNA control in the immune system: basic principles. Cell 136: 26-36, 2009.

19. Lu LF and Liston A: MicroRNA in the immune system, microRNA as an immune system. Immunology 127: 291-298, 2009.

20. O'Connell RM, Rao DS, Chaudhuri AA and Baltimore D: Physiological and pathological roles for microRNAs in the immune system. Nat Rev Immunol 10: 111-122, 2010.

21. Lu TX, Hartner J, Lim EJ, et al: MicroRNA-21 limits in vivo immune response-mediated activation of the IL-12/IFN-gamma pathway, Th1 polarization, and the severity of delayed-type hypersensitivity. J Immunol 187: 3362-3373, 2011.

22. Chen RF, Huang HC, Ou CY, et al: MicroRNA-21 expression in neonatal blood associated with antenatal immunoglobulin $\mathrm{E}$ production and development of allergic rhinitis. Clin Exp Allergy 40: 1482-1490, 2010.

23. Mattes J, Collison A, Plank M, Phipps S and Foster PS: Antagonism of microRNA-126 suppresses the effector function of TH2 cells and the development of allergic airways disease. Proc Natl Acad Sci USA 106: 18704-18709, 2009.

24. Chiba Y, Tanabe M, Goto K, Sakai H and Misawa M: Down-regulation of miR-133a contributes to up-regulation of Rhoa in bronchial smooth muscle cells. Am J Respir Crit Care Med 180: 713-719, 2009.

25. Chiba Y and Misawa M: MicroRNAs and their therapeutic potential for human diseases: miR-133a and bronchial smooth muscle hyperresponsiveness in asthma. J Pharmacol Sci 114: 264-268, 2010.

26. Collison A, Mattes J, Plank M and Foster PS: Inhibition of house dust mite-induced allergic airways disease by antagonism of microRNA-145 is comparable to glucocorticoid treatment. J Allergy Clin Immunol 128: 160-167, 2011.

27. Taganov KD, Boldin MP, Chang KJ and Baltimore D: NF-kappaB-dependent induction of microRNA miR-146, an inhibitor targeted to signaling proteins of innate immune responses. Proc Natl Acad Sci USA 103: 12481-12486, 2006.

28. Xiao C, Calado DP, Galler G, et al: MiR-150 controls B cell differentiation by targeting the transcription factor $\mathrm{c}-\mathrm{Myb}$ Cell 131: 146-159, 2007

29. Faraoni I, Antonetti FR, Cardone J and Bonmassar E: miR-155 gene: a typical multifunctional microRNA. Biochim Biophys Acta 1792: 497-505, 2009

30. Costinean S, Zanesi N, Pekarsky Y, et al: Pre-B cell proliferation and lymphoblastic leukemia/high-grade lymphoma in E(mu)-miR155 transgenic mice. Proc Natl Acad Sci USA 103: 7024-7029, 2006.

31. Wang P, Hou J, Lin L, et al: Inducible microRNA-155 feedback promotes type I IFN signaling in antiviral innate immunity by targeting suppressor of cytokine signaling 1 . J Immunol 185: 6226-6233, 2010

32. Lu LF, Thai TH, Calado DP, et al: Foxp3-dependent microRNA155 confers competitive fitness to regulatory $\mathrm{T}$ cells by targeting SOCS1 protein. Immunity 30: 80-91, 2009.

33. Evel-Kabler K, Song XT, Aldrich M, Huang XF and Chen SY: SOCS1 restricts dendritic cells' ability to break self tolerance and induce antitumor immunity by regulating IL-12 production and signaling. J Clin Invest 116: 90-100, 2006.

34. Rodriguez A, Vigorito E, Clare S, et al: Requirement of $\mathrm{bic} / \mathrm{microRNA}-155$ for normal immune function. Science 316 : 608-611, 2007

35. Harada M, Nakashima K, Hirota T, et al: Functional polymorphism in the suppressor of cytokine signaling 1 gene associated with adult asthma. Am J Respir Cell Mol Biol 36: 491-496, 2007 
36. Yoshimura A, Naka T and Kubo M: SOCS proteins, cytokine signaling and immune regulation. Nat Rev Immunol 7: 454-465, 2007.

37. Banerjee A, Schambach F, DeJong CS, Hammond SM and Reiner SL: Micro-RNA-155 inhibits IFN-gamma signaling in CD4 ${ }^{+}$T cells. Eur J Immunol 40: 225-231, 2010.

38. Li QJ, Chau J, Ebert PJ, et al: MiR-181a is an intrinsic modulator of T cell sensitivity and selection. Cell 129: 147-161, 2007.

39. Maes T, Tournoy KG and Joos GF: Gene therapy for allergic airway diseases. Curr Allergy Asthma Rep 11: 163-172, 2011.
40. Luo YY, Deng YQ and Tao ZZ: Expression of specific microRNA-135a in nasal mucosa of allergic rhinitis mice. Mil Med J S Chin 26: 102-105, 2012 (In Chinese).

41. Chakir H, Wang H, Lefebvre DE, Webb J and Scott FW: T-bet/GATA-3 ratio as a measure of the Th1/Th2 cytokine profile in mixed cell populations: predominant role of GATA-3. J Immunol Methods 278: 157-169, 2003. 